\title{
CORRECTIONS
}

\section{Thermodynamique des comportements rhéologiques en variables naturelles}

Rheol. Acta 22, 245-259 (1983)

C. Huet

Ecole Nationale des Ponts et Chaussées,

Centre d'Enseignement et de Recherches en Matériaux de Construction, Paris

1) Equations (7.4) et (7.5) du Tableau 7 page 255, lire:

$$
\begin{aligned}
& \frac{1}{T}=\frac{1}{T^{\mathrm{eq}}}(u, \Delta)=\frac{\partial s}{\partial u}, \\
& \frac{\Pi_{i j}}{\varrho_{0} T}=\frac{\Pi_{i j}^{\mathrm{eq}}}{\varrho_{0} T^{\mathrm{eq}}}(u, \Delta)=-\frac{\partial s}{\partial \Delta_{i j}} .
\end{aligned}
$$

2) Dans les équations (8.6) et (8.9) du Tableau 8, page 255 , au dernier membre à droite:

$$
\begin{aligned}
& \text { au lieu de } \frac{\Pi_{i j}^{\mathrm{eq}}}{\varrho_{0} T}, \text { lire } \frac{\Pi_{i j}^{\mathrm{eq}}}{\varrho_{0} T^{\mathrm{eq}}}, \\
& \text { au lieu de } \frac{\Pi_{i j}^{\mathrm{eq}}}{\varrho_{0} T} \dot{\Delta}_{i j} \text { lire } \frac{\Pi_{i j}^{\mathrm{eq}}}{\varrho_{0} T^{\mathrm{eq}}} \dot{\Delta}_{i j} .
\end{aligned}
$$

3) Equations (9.3) et (9.4) du Tableau 9, page 256, lire:

$$
\begin{aligned}
& \frac{1}{T}=\frac{1}{T^{\mathrm{eq}}}=\frac{\partial s^{\mathrm{eq}}}{\partial u}, \\
& \frac{\Pi_{i j}}{\varrho_{0} T}=\frac{\Pi_{i j}^{\mathrm{eq}}}{\varrho_{0} T^{\mathrm{eq}}}=-\frac{\partial s^{\mathrm{eq}}}{\partial \Delta_{i j}} .
\end{aligned}
$$

4) Page 241, 2e ligne sous le Tableau 4, au lieu de «nons», lire «nous».

Enfin, l'éditeur présente ses excuses pour les nombreuses fautes d'accents, de traits d'union et d'apostrophes commises, du fait d'une défaillance technique, au cours de l'impression à partir d'épreuves correctes de ce point de vue.

\section{(Reçu p.p. le 9 février 1984)}

Adresse de l'auteur:

Prof. C. Huet

CERMaC, ENPC,

28 , rue des Saints-Pères

F-75007 Paris

951

\section{ANNOUNCEMENTS}

\section{Symposium on Viscoelasticity and Rheology \\ October 16-18, 1984, Madison, Wisconsin (U.S.A.)}

The Mathematics Research Center at the University of Wisconsin-Madison will hold an Interdisciplinary Symposium on Viscoelasticity and Rheology in Madison on October 16-18, 1984. The Symposium will compare a diversity of views on constitutive theories, mathematical modelling, experimental and numerical methods, and comparisons between theoretical and experimental results. The 16 distinguished invited speakers (applied mathematicians, rheologists and mechanicians) are:
R. C. Armstrong, R. B. Bird, B. Coleman, C. Dafermos, H. Giesekus, O. Hassager, D. D. Joseph, A. S. Lodge, R. C. MacCamy, D. Malkus, M. Renardy, R. Tanner, E. Walsh, K. Walters, W. D. Williams, L. J. Zapas.

A detailed program will be available in August 1984, and further information can be obtained from

Mrs. Gladys Moran, Conference Secretary

Mathematics Research Center

University of Wisconsin-Madison

610 Walnut Street, WARF Bldg.

Madison, WI 53705 (U.S.A.)

Information can also be obtained from the organizers: John A. Nohel, Arthur S. Lodge and Michael Renardy.

Für die Schriftleitung verantwortlich: Prof. Dr. H. Giesekus, Abteilung Chemietechnik der Universität Dortmund, Postfach 500500, D-4600 Dortmund 50

Anzeigenverwaltung: Springer-Verlag GmbH \& Co. KG, Kurfürstendamm 237, 1000 Berlin 15. - Verantwortlich für den Anzeigenteil: E. Lückermann. - Verlag: Dr. Dietrich Steinkopff Verlag GmbH \& Co. KG, Postfach 111 008, D-6100 Darmstadt 11 - Gesamtherstellung: Graphischer Betrieb Konrad Triltsch, D-8700 Würzburg 